\title{
3.2 Reforma del plan de estudios de la carrera de medicina de la PUCMM: apuntes de una experiencia
}

\section{Resumen}

Este artículo relata los pasos que se siguieron en el proceso de mejorar el plan de estudios de la carrera de Medicina de la PUCMM. Se comienza con la justificación de dicho cambio, luego se sitúa la reforma realizada dentro del contexto universitario, nacional e internacional, y se termina señalando cuáles son los nuevos elementos introducidos. El artículo concluye enfatizando la importancia vital que tiene el seguimiento continuo a toda modificación curricular en una institución académica.

"Es tarea de la comunidad mantenerse siempre actualizada y más aún, adelantarse previsoramente, atisbando el porvenir y preparándose con la debida antelación a las exigencias de los tiempos y de las nuevas generaciones."

- Monseñor Agripino Núñez Collado, Realidad y Perspectivas de la Sociedad Dominicana

(2005)

"A menudo la escuela enseña contenidos del siglo XIX con profesores del Siglo XX a alumnos del siglo XX."

- Ezequiel Ander-Egg,

Debates y propuestas sobre la problemática educativa

Hemos estado varios años buscando soluciones para actualizar el plan de estudios de la carrera de Medicina de la Pontificia Universidad Católica Madre y Maestra (PUCMM), tanto desde la Facultad de Ciencias de la Salud como desde el Departamento de Medicina. En el año 2005, ocho profesores de Medicina realizaron la
Especialidad en Pedagogía Universitaria, auspiciada por el Programa de Superación del Profesorado de esta Universidad. Como resultado del impacto producido por los estudios de estos profesores, se llegó a un acuerdo con la Vicerrectoría Académica para realizar la reforma curricular. Para tales fines, se conformó un equipo integrado por varios profesores ${ }^{2}$, estudiantes, egresados y personal administrativo, con la participación de 2 asesores internacionales: el Dr. Carlos Alberto Brailovsky, de la Organización Panamericana de la Salud, y el Dr. Miguel Zabalza, invitado por el Programa de Superación del Profesorado.

Para la reforma curricular se utilizó la metodología del "Benchmarking", que según Spendolini (1992,p.15), es "el proceso sistemático y continuo para evaluar los productos, servicios y procesos de trabajo de las organizaciones que son reconocidas como representantes de la mejores prácticas, con el propósito de realizar mejoras organizacionales". Se revisaron más de 180 planes de estudio de Medicina de diferentes universidades de América y de Europa en relación a las asignaturas, número de créditos, estrategias de enseñanza, entre otros. En el 2004 se participó en el Seminario "La Formación del Médico y la Perspectiva del Desempeño Profesional", en la Universidad Autónoma de México, con la asistencia de invitados de Estados Unidos y Cánada, donde se evaluaron los programas de estudio de México. En este seminario estaban también representantes españoles de la Unión Europea, quienes expusieron las conclusiones sobre la

\footnotetext{
1 Doctor en Medicina, Especialista en Medicina Interna. Director del Departamento de Medicina, Pontificia Universidad Católica Madre y Maestra, Santiago de los Caballeros.

2 El equipo estaba compuesto por los doctores Luis José Castillo, José Joaquín Zouain, Oscar Madera, Félix Contreras, Ángela Matos, Sergio Díaz y Daniel Rivera.
} 
carrera de Medicina del Espacio Europeo de Educación Superior (EEES). En esta ocasión, se hizo contacto con uno de los mejores evaluadores de planes de estudio de educación médica, el Dr. José Venturelli, quien es profesor de Pediatría en la Universidad de McMaster, en Canadá, y autor del libro Educación Médica publicado por la Organización Panamericana de la Salud.

En un tiempo de más de 200 horas de trabajo, se realizó el diagnóstico del sistema modular de enseñanza que se había estado usando en la PUCMM. Se revisó el documento básico de Medicina del año 2002 y se realizaron cambios en la misión, la visión, el perfil del egresado, el macrocurrículo, el microcurrículo, las competencias y los objetivos del nuevo plan de estudios. Esto dio como resultado
3. Evaluaciones muy frecuentes (cada 15 días) y esto, junto con el poco tiempo para estudiar y la gran cantidad de material de estudio, impedía la asimilación de los conocimientos y la reflexión de los mismos.

4. Era necesario reformular los créditos de cada una de las asignaturas mediante el Reglamento Académico de la Universidad.

5. Se necesitaba hacer un nuevo plan de estudios que tuviera los diferentes componentes de los módulos con los mismos nombres y número de créditos, tanto a nivel nacional como internacional. Por ejemplo, el módulo que antes se llamaba "Diabetes" ahora recibe el nombre de "Endocrinología".

6. Se teníaun plan de estudios único, en el campus de Santiago, que no podía ser aplicado en el Recinto Santo Tomás de

"...se realizaron cambios en la misión, la visión, el perfil del egresado, el macrocurrículo, el microcurrículo, las competencias y los objetivos del nuevo plan de estudios".

el Pensum IV, que fue aprobado por el Consejo de la Facultad de Ciencias de la Salud, el Comité de Currículum y el Consejo Académico de la Universidad. Este nuevo pensum entró en vigencia en el semestre agosto - diciembre 2005, con 102 estudiantes nuevos.

\section{Justificación para la reforma curricular: evaluación del plan de estudios}

\section{a) En el contexto universitario:}

1. El sistema modular existente no estaba siendo implementado de forma integral y tenía mucha evaluación sumativa.

2. Había demasiadas horas de clases en las aulas, prácticas hospitalarias deficientes con horarios extensos y agotadores, que dejaban poco tiempo para el estudio.
Aquino, en Santo Domingo.

7. Las fechas de entrada y de salida de clases, así como las fechas de retiro y recuperación de asignaturas, eran diferentes al calendario académico del resto de las carreras que se ofrecían en la Universidad.

8. Si se mejoraba el índice relativo de las áreas biológica y clínica en un 0.5\% más, se evitaría que muchos estudiantes estuvieran en Prueba Académica y separados de la carrera, sólo con ese detalle.

9. Resultaba difícil homologar las asignaturas de la carrera atendiendo a los requisitos internacionales para ser fácilmente verificables por universidades y hospitales donde participan los egresados.

10. Los horarios de clases eran de siete de la mañana a ocho de la noche, quedando entre horarios espacios de hasta tres 
horas libres, y los exámenes sabatinos dejaban poco tiempo para la reflexión de los contenidos de las asignaturas.

11. Había desconocimiento pedagógico para el uso del sistema modular por gran parte de los profesores y éstos utilizaban la conferencia magistral como única forma de metodología de enseñanza.

12. Había falta de integración entre los profesores de las asignaturas teóricas y los profesores de la práctica clínica, con el desarrollo de los contenidos del plan de estudios.

13. Poca actualización de los nuevos contenidos temáticos.

14. Un programa docente centrado en el profesor.

15. Se determinó que se debía aumentar el número de créditos de las Ciencias Básicas o Preclínicas para mejorar los

\section{b) En el contexto nacional:}

1. La Secretaría de Estado de Educación Superior, Ciencia y Tecnología (SEESCYT) publicó en el 1996, mediante el Decreto 517-96, emitido por la Presidencia de la República, las normas para la evaluación y/o aprobación de escuelas de medicina en el país.

2. La Ley General de Salud (48-01) y la Ley de Seguridad Social (87-01) promulgadas el 9 de mayo del 2001.

3. Mayor emigración de los médicos dominicanos hacia Estados Unidos. Según Mullan (2005), hasta la fecha, de un total de 18,932 médicos egresados de universidades dominicanas, 3,262 emigraron a ejercer en Estados Unidos. Esto indica un factor de emigración de $17.2 \%$, en comparación con México, que de 177,007 médicos egresados sólo

\section{"Nos guiamos por el enfoque del nuevo docente: flexible, innovador,}

facilitador y tutor, así como las nuevas estrategias de formación con métodos integradores de teoría y práctica".

resultados académicos a nivel nacional e internacional.

16. Era necesario disminuir el número de fracasos o retiros del área biológica.

17. Se debía aumentar el número de estudiantes que entraban del Ciclo Básico a la carrera y el número de estudiantes que terminaban.

18. Se consideró pertinente disminuir el tiempo de duración de la carrera de Medicina de seis años y medio a cinco años y ocho meses, utilizando de manera regular todos los veranos. emigraron 4,741 médicos a los Estados Unidos, con un factor de emigración de $2.7 \%$.

\section{c) En el contexto internacional:}

1. La Universidad ha tomado en cuenta las nuevas regulaciones del Espacio Europeo de Educación Superior (EEES) (Benito \& Cruz, 2005), que proponen pensar las competencias en sentido transversal y para el mercado laboral. También los artículos 48, 49 y 89 de la Declaración de Guadalajara de la III Cumbre de Jefes de Estado de América Latina, el Caribe y la Unión Europea, en mayo de 2004, que tratan del proceso de acreditación y certificación.

2. Nos guiamos por el enfoque del nuevo docente: flexible, innovador, facilitador 
y tutor, así como las nuevas estrategias de formación con métodos integradores de teoría y práctica. La nueva educación médica está centrada en competencias del estudiante con evaluación formativa y sumativa. Se promueve la autonomía, la equidad y la justicia social y el aprendizaje en grupos pequeños basado en problemas y evidencias (Venturelli, 2003).

3. La incorporación de nuevos contenidos temáticos: Historia y Antropología Médica, Informática, Salud Sexual y Reproductiva, Medicina Laboral y Ambiental, Bioética, Medicina Familiar, Imagenología, Geriatría, Medicina Legal.

4. Los cambios en los enfoques educativos: educación de adultos, desarrollo de destrezas de aprendizaje para toda la vida, integración entre las Ciencias nicas tomando en cuenta el examen Step 1 del USMLE para dominicanos y extranjeros.

\section{Innovaciones del nuevo plan de estudios de Medicina}

1. Un nuevo documento básico de la carrera de Medicina donde se presenta la misión, la visión, el perfil del egresado, los valores, las competencias y los objetivos mínimos de la formación, acorde con las exigencias del profesional en el siglosiglo XXI.

2. Un plan de estudios compuesto por 13 unidades distribuidas en períodos de 14 semanas, donde lo que era antes un verano de 10 semanas se convierte en un período como los otros, de 14 semanas. Duración de la carrera: 5 años y 8 meses, 358 créditos y 13 unidades.

\section{"Se normaliza la carrera de Medicina acorde con las reglamentaciones del calendario académico y se fijan con más claridad los créditos de las asigna- turas para que los procesos administrativos se descentralicen, beneficiando así al estudiante y al Departamento de Medicina".}

Básicas, Preclínicas y Clínicas, así como nuevas destrezas para la adquisición de información. Capacidad de evaluación crítica ante terapias, nuevos conocimientos y organización del proceso.

5. La necesidad de mejorar los niveles de puntuación en los exámenes de reválida de Puerto Rico y del United State Medical Licensing Examination (USMLE) de Estados Unidos.

6. Facilitar la homologación y acreditación de los egresados de Medicina que van a realizar especialidades en Estados Unidos, México y Europa, conteniendo créditos y nombres de las asignaturas similares a la mayoría de los países con escuelas de Medicina.

7. Facilitar que los dos primeros años de la carrera de Medicina estén concentrados en las Ciencias Básicas y Preclí-
3. Se estructuró un macrocurrículo donde las 6 primeras unidades concentran las asignaturas de Ciencias Básicas y Preclínicas favoreciendo que puedan completar la primera etapa de su formación y pueda ser evaluada en los exámenes internacionales (Step 1). Luego 7 unidades más de asignaturas de Clínica e Internado Rotatorio, pudiendo al final del mismo examinarse de la 2da. parte clínica a nivel de exámenes internacionales (Step 2) .

4. La relación de las Ciencias Básicas con las asignaturas de Clínica se realiza de forma gradual y están unidas en las 13 unidades mediante 3 ejes transversales: Profesión Médica, Investigación y Eje Social. 
5. Se ofrece por primera vez una asignatura electiva de la Carrera de Medicina de 3 créditos $y$, otra, donde se pueda elegir entre las áreas de Bioética y Medicina Basada en Evidencias.

6. Horarios más adecuados al estudiante: tanda matutina, de 7:00 a. m. a 1:00 p. m.; $\mathrm{y}$ tanda vespertina, que se ofrece una vez al año en el tercer período de cada año académico en el horario de 2:00 p. m. a 8:00 p. m. Además, tres entradas por año de forma regular: agosto, enero y mayo.

7. Se normaliza la carrera de Medicina acorde con las reglamentaciones del calendario académico y se fijan con más claridad los créditos de las asignaturas para que los procesos administrativos se descentralicen, beneficiando así al estudiante y al Departamento de Medicina. Por ejemplo, los retiros, los períodos de exámenes finales y la publicación de notas con 48 créditos. Las Preclínicas, de 45 créditos, donde entran los principios básicos del proceso patológico, y luego, el bloque Clínico con 133 créditos, donde se ha preservado la integración teórico-práctica en las Salas Clínicas Hospitalarias, igual que el Plan de Estudios anterior. También se ha preservado el área Social con 15 créditos y el área de Investigación con 20 créditos.

10. El Internado Rotatorio tiene 3 asignaturas teórico-prácticas y de discusión como: Emergencias Médicas, Medicina Crítica y Grandes Temas de la Medicina. Se amplió el Internado Social y la rotación en hospitales extranjerosextranjeros en el área de Medicina Interna.

11. Mejor distribución de las asignaturas. Ahora no se imparten por semanas como el caso de las Clínicas. Anteriormente eran de 4 semanas con exámenes cada 15 días y

"Se ha creado un sistema de tutorías donde cada profesor de tiempo completo y medio tiempo recibe 5 estudiantes por cada 9 estudiantes que ingresen. El profesor será asesor, orientador y dará seguimiento al desempeño académico de los alumnos".

se realizan según el calendario.

8. Los créditos, los nombres y la ubicación de las asignaturas en el macrocurrículo son ahora similares a los formatos de Estados Unidos, el EEES (Benito \& Cruz, 2005) y de la mayoría de los países que tienen carrera de Medicina, donde podemos homologar nuestro plan de estudios en los ámbitos nacional e internacional. Se ha facilitado la tarea al Departamento de Admisiones en relación a las transferencias y convalidaciones con la mayoría de países que tienen carrera de Medicina o Bachillerato en Ciencias.

9. La distribución de las asignaturas y los contenidos de éstas se realizan con un enfoque similar al esquema actual de formación de la mayoría de las universidades. El ciclo Básico o Premédica de 90 créditos, las Ciencias Básicas normales muchos contenidos, lo cual no permitía la reflexión y sedimentación de los conocimientos. En el pensum actual se imparten a lo largo de 14 semanas. Esto permite tanto al docente como al educando preparar mejor el material didáctico y colocar los contenidos en la página electrónica para permitir la consulta de los estudiantes y la asesoría del profesor.

12. Se ha creado un sistema de tutorías donde cada profesor de tiempo completo y medio tiempo recibe 5 estudiantes por cada 9 estudiantes que ingresen. El profesor será asesor, orientador y dará seguimiento al desempeño académico de los alumnos.

13. Se ha activado la Unidad de Educación, la Coordinación Académica, y se han creado los puestos de Coordinadores de Unidades Docentes. 
14. Se han creado nuevos métodos de evaluación: por objetivos, competencias, habilidades, destrezas y contenidos de las asignaturas, de acuerdo al perfil del egresado y objetivos del médico del siglo XXI.

15. Se han reforzado, desde el inicio de la carrera, el aprender a aprender, el manual de la Información Biomédica, la co-evaluación y la autoevaluación.

16. Hay un mayor control del currículo oficial y la eliminación de lo que Gimeno \& Pérez (2002, pp. 152-153) denominan "currículum oculto", que se había creado en los últimos 15 años.

17. Un sistema integrado y modular con concentración de materias en un ciclo de Ciencias Básicas, dividido en dos bloques: Estructura y Función Normal del Cuerpo Humano e Introducción a la Enfermedad. Un segundo ciclo de Ciencias Clínicas integrado por dos bloques:Clínico e Internado.

Queremos puntualizar que luego de realizada la reforma curricular es cuando comienza el verdadero trabajo. Se hace necesario en su implementación el seguimiento constante de muchos detalles, tales como evitar las distracciones y los acomodamientos, estar atentos a la resistencia al cambio, mantener las reuniones periódicas de la Coordinación Académica del Departamento de Medicina y hacer evaluaciones del proceso. Además, continuar con la capacitación de profesores a través del PSP, mantener el canal abierto con los estudiantes mediante tutorías, evaluar el rendimiento académico, ver los indicadores básicos de las asignaturas: número de retirados, número de fracasos, quiénes están acordes con el pensum, entre otros.

Es preciso utilizar mejores estrategias didácticas, manteniendo el control sobre los contenidos, nuevos textos acordes con las nuevas competencias, promover más participación activa de los discentes, hacer evaluaciones periódicas. En definitiva, que el escenario del aprendizaje -el aula, el laboratorio, el hospital -, sea un lugar donde se construya conocimiento y que el profesor sea el líder del proceso en la formación del médico para el siglo XXI. *

\section{Referencias bibliográficas}

Ander-Egg, E., (2005). Debates y propuestas sobre la problemática educativa. Algunas reflexiones sobre los retos del futuro Inmediato. Rosario, Argentina: Homo Sapiens.

Benito, A. \& Cruz, A. , (2005). Nuevas claves para la docencia universitaria en el Espacio Europeo de Educación Superior. Madrid: Narcea.

Gimeno, J. \& Pérez, A. (2002). Comprender y transformar la enseñanza. Madrid: Morata.

Mullan, F, (2005). The Metrics of the Physician Brain Drain. The New England Journal of Medicine, 353 (17), 1810-1917.

Núñez, A., (2005). Cambios y Tendencias en la Educación Superior. En: Realidad y Perspectivas de la Sociedad Dominicana (pp. 553-554). Santo Domingo: Pontificia Universidad Católica Madre y Maestra

Declaración de Guadalajara. (2004, 28 y 29 de mayo). Guadalajara: III Cumbre América Latina y el Caribe-Unión Europea. Extraído el 5 de mayo de 2006, de http://www.oei.es/guadalajara.pdf

Venturelli, J., (2003). Educación Médica. Washington: Organización Panamericana de la Salud.

Spendolini, M. (1992). Benchmarking. Bogotá: Editorial Grupo Norma. 